\title{
Ne pas évaluer l'asthme : une erreur
}

$\mathrm{S}$ elon les statistiques nationales et mondiales sur la santé, l'asthme a atteint des proportions d'épidémie. Les données épidémiologiques les plus récentes révèlent en effet que 300 millions de personnes dans le monde souffrent d'asthme; le fardeau mondial augmentera vraisemblablement de 100 millions de cas d'ici à 2025․ Certaines données indiquent toutefois que l'on surestime peut-être l'ordre de grandeur du problème. Dans ce numéro du $J A M C^{2}$, Aaron et ses collaborateurs montrent que dans une cohorte raisonnablement représentative de la population canadienne, recrutée de façon prospective, près du tiers des patients chez lesquels on a diagnostiqué la présence de l'asthme ne montrent en réalité aucun signe de la maladie après avoir subi des tests rigoureux et cessé de prendre des médicaments contre l'asthme.

Pour comprendre ces résultats dans le bon contexte, il ne faut pas oublier l'origine de notre approche clinique de l'asthme. L'asthme peut tuer, mais presque tous les décès causés par l'asthme peuvent être évités. La mortalité attribuable à l'asthme a diminué de plus de $70 \%$ au Canada depuis 20 ans et nous affichons maintenant un des taux de mortalité attribuable à l'asthme les plus faibles au monde' ${ }^{1}$. Pourtant, 287 Canadiens sont morts de l'asthme en $2003^{3}$. Ces décès, fort probablement, auraient pu être évités. L'asthme cause aussi un fardeau important d'incapacité, dont l'ordre de grandeur ressemble à celui du diabète'. Une majorité des Canadiens qui souffrent d'asthme n'ont pas encore réussi à contrôler la maladie ${ }^{4}$, même s'il existe beaucoup de médicaments antiasthmatiques très efficaces qui devraient permettre à la plupart des patients de bien maitriser leur asthme tout le temps ou presque. Cette situation est attribuable en grande partie au fait que la consommation de médicaments antiasthmatiques demeure très inférieure au niveau recommandé par les guides factuels ${ }^{5}$.

Il ne faut donc pas interpréter les constatations d'Aaron et ses collaborateurs comme une raison de moins soupçonner l'asthme face à des patients qui présentent des symptômes respiratoires inexpliqués. Les auteurs décrivent plutôt un problème important dans notre système de traitement de l'asthme : au lieu d'être simplement surdiagnostiqué, l'asthme est mal diagnostiqué. Le diagnostic erroné d'asthme chez des patients qui présentent des symptômes persistants ou répétitifs a des répercussions importantes qui peuvent être sérieuses à la fois pour les patients et pour le système de santé. Des symptômes que l'on suppose attribuables à l'asthme peuvent indiquer un autre problème médical, qui demeure ainsi non diagnostiqué ni traité. Par exemple, on confond régulièrement, sur le plan clinique, l'asthme et la maladie pulmonaire chronique obstructive chez les adultes. Les examens objectifs offrent le seul moyen fiable de distinguer les deux maladies ${ }^{6}$ dans ces cas. Chez les enfants de 6 ans ou plus, les tests peuvent permettre de distinguer l'asthme des symptômes d'infections à répétition attribuables à une immunodéficience ou à la fibrose kystique. À l'inverse, des patients dont les symptômes d'asthme sont atypiques ou mal perçus risquent de recevoir un traitement insuffisant si on les évalue sans examen objectif. Or, ces patients sont souvent ceux qui risquent le plus d'avoir une maladie instable. Et comme l'asthme est une maladie chronique, les conséquences d'un mauvais diagnostic peuvent durer des années.

La fréquence du mauvais diagnostic est particulièrement frappante puisque des mesures objectives de la fonction pulmonaire permettent de diagnostiquer avec précision la plupart des cas d'asthme. La forme la plus élémentaire de ces tests est la spirométrie, qui mesure le débit et le volume respiratoires des poumons lorsqu'on demande au patient d'expirer totalement aussi rapidement que possible. Une diminution du débit indique la présence d'une maladie pulmonaire obstructive, dont l'asthme est de loin la plus répandue. Une amélioration du débit après administration d'un bronchodilatateur à action rapide confirme le diagnostic d'asthme. Même si les laboratoires d'analyse de la fonction pulmonaire administrent des examens plus poussés au moyen d'un matériel coûteux et complexe, il est possible de procéder à une spirométrie avec une exactitude acceptable en utilisant du matériel portatif que l'on peut installer dans un cabinet de médecin moyennant un investissement de départ de quelques milliers de dollars.

Pourtant, presque la moitié des patients souffrant d'asthme diagnostiqué par un médecin n'ont jamais subi une spirométrie ${ }^{4}$. Beaucoup d'autres n'ont jamais subi les examens plus précis nécessaires pour distinguer l'asthme d'autres pneumopathies obstructives. Sans ces examens, un diagnostic clinique d'asthme, même posé par des spécialistes de l'asthme, est erroné chez environ le tiers des patients ${ }^{6}$ parce que les symptômes de l'asthme ressemblent à ceux de beaucoup d'autres problèmes respiratoires. Comme la grande majorité des patients qui souffrent d'asthme sont diagnostiqués et pris en charge par leur médecin de famille, l'obligation d'assurer l'exactitude du diagnostic incombe inévitablement surtout aux médecins de première ligne.

Il est inacceptable de ne pas diagnostiquer l'asthme objectivement. On considérerait qu'un médecin qui tenterait de traiter l'hypertension sans prendre la tension artérielle ou l'hypercholestérolémie sans mesurer les taux de cholestérol sérique ne se conforme pas à une norme de soin adéquate. Traiter l'asthme sans avoir procédé au moins à une spirométrie, c'est la même chose. Il faut beaucoup plus de connaissances spécialisées pour interpréter des résultats de spirométrie que pour utiliser un sphygmomanomètre ou prescrire une analyse sanguine; de même, la lecture d'un électrocardiogramme exige des connaissances et de la formation, mais c'est pourtant un examen de routine pour tout médecin de première ligne. Rien ne peut justifier que la spirométrie ne soit pas tout aussi routinière dans une pratique de soins primaires. Les médecins qui n'utilisent pas la spirométrie pour leurs patients asthmatiques ne devraient pas traiter l'asthme. Or, les médecins de première ligne ne peuvent éviter de prendre en charge cette maladie très courante.

Il existe des obstacles à l'administration des tests de fonction pulmonaire. L'accès aux laboratoires qui effectuent ces tests est limité. Les gouvernements provinciaux limitent en fait le nombre 
Encadré 1 : Comment surmonter les obstacles pour obtenir un test de fonction pulmonaire

- Savoir où se trouvent les laboratoires locaux de fonction pulmonaire et connaître la longueur des listes dattente.

- Les patients sont plus susceptibles de se soumettre à un examen s'ils prennent rendez-vous à un laboratoire de fonction pulmonaire pendant qu'ils se trouvent à votre bureau.

- Demandez aux laboratoires de vous télécopier les résultats non interprétés afin que vous puissiez commencer à les interpréter sans avoir à attendre le rapport officiel.

- La participation à un réseau de mentorat où les médecins de première ligne ont accès à un collègue expert qui les aide à interpréter des résultats difficiles de spirométrie peut constituer un outil de soutien utile.

- On peut renforcer ses compétences en techniques de test en participant à des programmes d'éducation continue.

de permis, ce qui empêche généralement de nouveaux laboratoires d'ouvrir leurs portes. Comme beaucoup d'autres ressources en soins de santé, l'accès aux tests de fonction pulmonaire varie aussi selon la région géographique. Ainsi, les laboratoires d'hôpitaux ont souvent de longues listes d'attente même si quelques-uns offrent des tests de spirométrie sans rendez-vous. Même après le test, le temps nécessaire à l'interprétation peut retarder la transmission des résultats au médecin traitant.

C'est pourquoi, afin de veiller à ce que les patients obtiennent des soins adéquats en temps opportun, les médecins de première ligne ne peuvent compter exclusivement sur les laboratoires de soins tertiaires administrés par des spécialistes pour obtenir des services de spirométrie. La plupart devraient plutôt offrir les tests dans leur propre cabinet. Même s'il est tout à fait possible pour les médecins de première ligne d'offrir les tests de spirométrie en service externe ${ }^{7}$, l'équipement exige un investissement initial, il faut le calibrer périodiquement et suivre une formation pour administrer le test et en interpréter les résultats. De plus, le remboursement des tests de spirométrie varie selon la province : le Québec, la Nouvelle-Écosse et Terre-Neuve-et-Labrador ne prévoient aucun financement des tests de spirométrie en soins primaires administrés en cabinet et la Colombie-Britannique ne paiera pas une spirométrie pratiquée le jour même d'une consultation en cabinet de soins primaires pour un cas d'asthme.

$\mathrm{Au}$ lieu de leur faire passer un examen, les médecins traitent souvent les patients qui ont des symptômes respiratoires de façon empirique et indéfiniment. C'est plus facile, mais ce n'est peut-être pas de la bonne médecine. Beaucoup de ces patients sont exposés inutilement à des réactions indésirables possibles (quoique le plus souvent mineures) et à des coûts qui peuvent dépasser 100 \$ par mois pour un seul médicament. Même s'il peut être rationnel d'administrer un traitement à l'essai comme moyen de confirmer un diagnostic d'asthme, il faut mesurer objectivement la réaction au traitement, sinon ce type d'essai aboutira à la fois à des diagnostics insuffisants et à des diagnostics excessifs.

Certains médecins recommandent que leurs patients surveil- lent leur débit expiratoire maximal à la maison pour remplacer la spirométrie. Même s'il est parfois possible de confirmer ainsi le diagnostic d'asthme, la mesure du débit maximal constitue un substitut inadéquat parce qu'elle est beaucoup plus grossière et moins précise que la spirométrie. La mesure du débit maximal a un rôle à jouer chez certains patients dans l'autosurveillance et la détermination d'expositions professionnelles qui provoquent l'asthme, mais elle ne peut remplacer la spirométrie comme outil de pratique.

Il faut au moins confirmer par spirométrie un diagnostic d'asthme soupçonné. Il faut au besoin faire subir à ces patients des tests plus spécialisés comme une provocation à la méthacholine. Pour satisfaire à cette norme de soin, tous les médecins qui traitent l'asthme doivent référer régulièrement les patients à un laboratoire de fonction pulmonaire, avoir dans leur propre clinique du personnel pour administrer une spirométrie ou le faire eux-mêmes. Il faut surmonter les obstacles à cet égard et il est possible de le faire (Encadré 1). Par ailleurs, les gouvernements doivent intervenir et investir dans la prise en charge appropriée de l'asthme, notamment autoriser davantage de laboratoires de fonction pulmonaire afin que les tests appropriés soient disponibles — et remboursés dans tout milieu de pratique partout au Canada. Enfin, les patients chez qui on a diagnostiqué l'asthme mais qui n'ont pas subi une spirométrie au moins une fois devraient exiger que leur médecin leur dise pourquoi ils n'ont pas subi ce test.

\section{Matthew B. Stanbrook MD PhD}

Rédacteur adjoint, Sciences

$J A M C$

\section{Alan Kaplan MD}

Président

Association canadienne des médecins de famille contre

l'asthme

Edmonton (Alb.)

Avec l'équipe de rédaction de l'éditorial (Paul C. Hébert MD MHSc, Barbara Sibbald BJ, Ken Flegel MDCM MSc,

Noni MacDonald MD MSc et Amir Attaran LLB DPhil).

Intérêts concurrents: Aucuns déclarés pour Alan Kaplan. Voir www.cmaj.ca/misc/edboard.shtml pour les déclarations de l'équipe de rédaction de l'éditorial.

Traduit par le Service de traduction de l'AMC.

\section{RÉFÉRENCES}

1. Masoli M, Fabian D, Holt S, et al. Global burden of asthma. Global Initiative for Asthma (GINA); 2004. Disponible : www.ginasthma.com/ReportItem .asp?11=2\&12=2\&intId=94 (consulté le 14 octobre 2008).

2. Aaron SD, Vandemheen KL, Boulet LP, et al. Overdiagnosis of asthma in obese and non-obese adults. CMAJ 2008;179:1121-31.

3. Santé Canada. Votre santé et vous. Asthme. Ottawa (Ont.) : Santé Canada; 2006 Disponible : www.hc-sc.gc.ca/hl-vs/alt_formats/pacrb-dgapcr/pdf/iyh-vsv/diseases-maladies/asthm-fra.pdf (consulté le 14 octobre 2008).

4. Chapman KR, Boulet L-P, Rea RM, et al. Suboptimal asthma control: prevalence, detection and consequences in general practice. Eur Respir J 2008;31:320-5.

5. Klomp H, Lawson JA, Cockcroft DW, et al. Examining asthma quality of care using a population-based approach. CMAJ 2008;178:1013-21.

6. Tinkelman DG, Price DB, Nordyke RJ, et al. Misdiagnosis of COPD and asthma in primary care patients 40 years of age and over. $J$ Asthma 2006;43:75-80.

7. Yawn BP, Enright PL, Lemanske RF Jr, et al. Spirometry can be done in family physicians' offices and alters clinical decisions in management of asthma and COPD. Chest 2007;132:1162-8. 